\title{
REAVI
}

\section{UMA ANÁLISE SOBRE O SISTEMA NACIONAL DE INOVAÇÃO DO BRASIL}

\author{
Guilherme Paraol de Matos ${ }^{1}$, Clarissa Stefani Teixeira ${ }^{2}$ \\ ${ }^{1}$ gparaol@gmail.com, ${ }^{2}$ Clastefaniegmail.com
}

\begin{abstract}
Resumo
O sistema de inovação do Brasil é constituído por diversos atores. Dessa forma, o objetivo do presente artigo é apresentar as instituições e fluxos de interações presentes no sistema brasileiro de inovação. Para tanto, utiliza-se de uma revisão bibliográfica e documental. Como resultado, pode-se compreender que existem diversas instituições no país que corroboram para a $\mathrm{C}, \mathrm{T} \& \mathrm{I}$ nacional, onde o maior fluxo de interação está presente entre governo (fomento), grandes empresas e ICT's. Por fim, cabe destacar que o Brasil ainda possui um longo caminho até alcançar economias desenvolvidas.
\end{abstract}

Palavras-chave: Sistema de inovação. Brasil. C\&T.

\section{AN ANALYSIS ON BRAZIL'S NATIONAL INNOVATION SYSTEM}

\begin{abstract}
In Brazil's innovation system, there are several actors. Thus, the objective of this paper is to present the institutions and interaction flows present in the Brazilian innovation system. To this end, we use a bibliographic and documentary review. As a result, it can be understood that there are several institutions in the country that corroborate the national $C, T \& I$, where the greatest flow of interaction is present between government (promotion), large companies and ICT's. Finally, be noted that Brazil still has a long way to go to developed economies.
\end{abstract}

Keywords: Innovation system. Brazil. $S \& T$.

\section{Introdução}

Na década de 80 e 90 foi criado o conceito de sistemas de inovação por meio de um projeto da Organização para a Cooperação e Desenvolvimento Econômico (OCDE) (ASHEIM; GRILLITSCH; TRIPPL, 2015), e desenvolvido posteriormente por Lundvall (1992), Nelson (1993) e Freeman (1995). Tal abordagem foi inspirada na teoria institucional sistêmica, o qual considera a inovação resultado de processos de conhecimento e aprendizagem complexos, interativos e cumulativos, nos quais participam diversos atores (ASHEIM; SMITH; OUGHTON, 2011; TANG et al., 2015; ASHEIM; GRILLITSCH; TRIPPL, 2015). O foco dessa abordagem são as instituições e suas relações, que devem ser fortalecidas para criar um sistema de inovação maduro (LARANJA et al., 2008).

A abordagem de sistema nacional de inovação (SNI), e posteriormente, de sistemas regionais de inovação (COOKE, 1992), é a teoria que mais influenciou políticas de ciência e tecnologia (C\&T) no mundo (LARANJA et al., 2008). Casos de aplicação dessa teoria em políticas públicas podem ser visualizadas mais recentemente na União Europeia, chamada de estratégia

Revista Eletrônica do Alto Vale do Itajaí - REAVI, v.08, n 13, p. 073-083, dez 2019 ISSN: 23164190, DOI 10.5965/2316419008112019073 


\section{REAVI}

regional de inovação, que visa desenvolver os sistemas regionais de inovação dos seus países membros (LARANJA et al., 2008).

No Brasil os primeiros passos para o desenvolvimento da Ciência, Tecnologia e Inovação (C,T\&I) foram dados ainda no final do século XIX, a partir da criação das primeiras instituições de pesquisa. Porém, é considerado o marco inicial do Sistema Nacional de Inovação do Brasil a criação da Campanha de Aperfeiçoamento de Pessoal de Nível Superior (CAPES) e do Conselho Nacional de Pesquisas (CNPq), na década de 50 (FREITAS, 2014). Desde então, diversas políticas, instituições e instrumentos foram criados para incentivar a pesquisa, a tecnologia e a inovação por meio de incentivos fiscais, financiamentos e aportes financeiros reembolsáveis e não reembolsáveis.

As trajetórias dos SNIs estão diretamente relacionadas com as estratégias de desenvolvimento que cada país adota. Assim, os governos nacionais são protagonistas na articulação dos elementos constituintes de cada sistema. Desse modo, a trajetória de evolução do sistema de inovação brasileiro é marcada pela necessidade de emparelhamento do país com os sistemas mais avançados do mundo. Para tanto, diversas instituições foram criadas e investimentos realizados para isso. Nesse contexto, os principais atores desse sistema são as Instituições de Ciência, Tecnologia e Inovação (ICTs), as entidades da gestão pública e as empresas (BRASIL, 2016).

Dessa forma, o presente artigo tem como objetivo, apresentar a evolução do sistema de inovação brasileiro, sua configuração atual e suas interações. Para tanto, além da presente introdução, a seção 2 apresenta o referencial teórico; a seção 3 os métodos utilizados; a seção 4 os resultados encontrados e a seção 5 conclui o trabalho.

\section{Sistema de inovação}

A abordagem de Sistema de Inovação surgiu num projeto da OCDE sobre Ciência, Tecnologia e Competitividade no início dos anos 80. O projeto teve como premissa promover a competitividade na economia do conhecimento baseado em inovação (ASHEIM; GRILLITSCH; TRIPPL, 2015). A inovação na teoria neoclássica era considerada como um processo linear (entrada de pesquisa e desenvolvimento $(\mathrm{P} \& \mathrm{D})$ e saída do produto final), realizada por uma única empresa. Por sua vez, a teoria institucional sistêmica descreve a inovação como resultado da interação de diversos atores, com ênfase, principalmente, na atuação das instituições (LARANJA et al., 2008). Esta abordagem reconhece que a inovação normalmente resulta de processos de conhecimento e aprendizagem complexos, interativos e cumulativos, nos quais participam diversos atores (ASHEIM; SMITH; OUGHTON, 2011; TANG et al., 2015; ASHEIM; GRILLITSCH; TRIPPL, 2015).

Assim, a inovação começou a ser considerada resultado de um sistema, que envolve diferentes partes inter-relacionadas, no qual, estão presentes diversos atos criativos, da pesquisa ao serviço, agindo juntos de forma integrada em direção a um objetivo comum (GODIN, 2015). Em outras palavras, a percepção inicial de que a inovação é basicamente um ato individual de aprendizado, feito por uma empresa ou empresário, se expandiu para incluir o sistema maior (que consiste em várias instituições, estrutura de políticas, incentivos, etc.) (TANG et al., 2015; ASHEIM; GRILLITSCH; TRIPPL, 2015).

A abordagem de Sistema de Inovação reconhece, assim, que a inovação surge de múltiplas formas e resulta de interdependências entre uma variedade de atores. Esta nova compreensão da inovação teve profundas implicações políticas, sendo uma das abordagens mais utilizadas pelos Revista Eletrônica do Alto Vale do Itajaí - REAVI, v.08, n 13, p. 073-083, dez 2019 ISSN: 23164190, DOI 10.5965/2316419008112019073 


\section{REAVI}

formuladores de política até hoje (ASHEIM; SMITH; OUGHTON, 2011). De fato, nesta abordagem, a política de inovação deve apoiar a circulação de conhecimentos entre todos os atores envolvidos na geração de inovações. Assim, a atenção política se concentra em construir e apoiar às redes entre universidades, organizações de pesquisa, empresas e demais instituições em que essas interações são incorporadas (ASHEIM; GRILLITSCH; TRIPPL, 2015).

Porém, um Sistema de Inovação pode não gerar resultados quando as instituições se mostram ineficazes. Por exemplo, Zaraychenko et al. (2016), revelam que um ambiente que não corrobora com a colaboração das instituições e a realização de parcerias vantajosas para ambos torna o sistema engessado, dificultando suas ações. Questões jurídicas que não oferecem segurança para parceiras, principalmente, públicas e privadas, proteção ao desenvolvimento de patentes e a falta de atuação de instituições chaves, tornam-se impeditivos de um sistema capaz de gerar ciclos viciosos de inovação e retorno de investimentos. Assim, o objetivo da seção 4 é apresentar as políticas, instituições e interações do sistema de inovação brasileiro.

\section{Metodologia}

O trabalho foi desenvolvido por meio de uma pesquisa qualitativa, bibliográfica e documental. A pesquisa qualitativa compreende um conjunto de técnicas interpretativas que visam descrever e a decodificar os componentes de um sistema complexo de significados (LAKATOS, 2010). A pesquisa bibliográfica abrange toda obra científica já tornada pública em relação ao tema de estudo, desde publicações avulsas, boletins, jornais, revistas, livros, pesquisa, monografias, teses, etc. Sua finalidade é colocar o pesquisador em contato direto com tudo o que foi escrito, dito ou filmado sobre determinado assunto (LAKATOS, 2010). A análise documental constitui uma técnica importante na pesquisa qualitativa, seja complementando informações obtidas por outras técnicas, seja desvelando aspectos novos de um tema ou problema (LUDKE; ANDRÉ, 1986).

\section{Resultados e discussão}

\subsection{Os passos do Brasil na construção do seu sistema de inovação}

O sistema de inovação do Brasil é recente quando comparado aos países desenvolvidos. Mesmo assim, Freitas (2014) revela que as primeiras instituições com cunho científico e tecnológico foram criadas no final do século XIX. São estas, a Escola de Minas de Ouro Preto; o Instituto Agronômico de Campinas em 1887; e, o Instituto Butantã em 1899. No início do século XX, outras três instituições foram criadas, o Instituto Oswaldo Cruz, em 1901; a Academia Brasileira de Ciências em 1916 e, sobretudo, o surgimento da primeira Universidade moderna, a Universidade de São Paulo (USP), em 1934.

Porém, é a partir da década de 50 que a estruturação e modernização do sistema de inovação brasileiro começa a tomar forma. Não apenas por meio do surgimento de instituições, mas também, pela criação de políticas públicas, fundos de recursos e programas de fomento à C,T\&I. Assim, em 1951 é criado o CNPq (CNPq, 2019) e a CAPES (CAPES, 2019). De fato, as duas principais instituições que fomentam a ciência no Brasil até hoje, uma vez que, o CNPq fomenta a pesquisa científica e tecnológica e incentiva a formação de pesquisadores brasileiros (CNPq, 2019). Por sua vez, a CAPES assegura a existência de pessoal especializado, em quantidade e qualidade, suficientes para atender às necessidades dos empreendimentos públicos e privados, que visam ao desenvolvimento do país (CAPES, 2019).

Revista Eletrônica do Alto Vale do Itajaí - REAVI, v.08, n 13, p. 073-083, dez 2019 ISSN: 23164190, DOI 10.5965/2316419008112019073 


\section{REAVI}

Na década seguinte, a necessidade e a importância da pesquisa científica ganham relevância no âmbito dos governos federal e estaduais. Dessa forma, o Ministério da Educação e Cultura cria a Comissão Supervisora do Planos dos Institutos (COSUPI) e o Programa de Expansão Tecnológica (PROTEC) (CNPq, 2019). Em 1964 é criado o Fundo de Desenvolvimento Técnico Científico (FUNTEC) (FREITAS, 2014). Nesse ínterim, um marco importante para a ciência é o início da pós-graduação no Brasil, em 1965, quando é regulamentada e estabelecido conceitos e bases legais. Assim, neste ano foram classificados 27 cursos de mestrado e 11 de doutorado, totalizando 38 no país (CNPq, 2019). Em 1967, a Financiadoras de Estudos e Projetos (FINEP) é fundada, e, posteriormente, passa a operacionalizar o Fundo Nacional de Desenvolvimento Científico e Tecnológico (FNDCT) criado em 1969 (FINEP, 2019).

Estas instituições representam um marco para o desenvolvimento cientifico e tecnológico do Brasil, desde então. A FINEP, por exemplo, é o principal órgão de fomento a inovação no país. Sua missão é promover o desenvolvimento econômico e social do Brasil por meio do fomento público à C,T\&I em empresas, universidades, institutos tecnológicos e outras instituições públicas ou privadas. Portanto, atua em toda a cadeia da inovação, com foco em ações estratégicas, estruturantes e de impacto para o desenvolvimento sustentável do Brasil (FINEP, 2019b). Por sua vez, o FNDCT, operacionalizado pela FINEP desde 1971, foi criado para oferecer apoio financeiro aos programas e projetos prioritários de desenvolvimento científico e tecnológico destinado a financiar a expansão e consolidação do sistema de ciência e tecnologia do país (CNPq, 2019, FINEP, 2019). Assim, é um fundo de natureza contábil que tem como objetivo financiar a inovação e o desenvolvimento científico e tecnológico, com vistas a promover o desenvolvimento econômico e social do País (FINEP, 2019b). Na época, o FNDCT também atrelou um maior envolvimento do Banco Nacional de Desenvolvimento Econômico (BNDE) e do Ministério do Planejamento em relação ao fomento de recursos para a área de C,T\&I. A criação do fundo permitiu um grande salto de investimentos para a área, sendo responsável por alavancar a implementação de parques de pesquisas científicos no país (BALBACHEVSKY, 2010; FREITAS, 2014; OLIVEIRA, 2016).

Ainda, no âmbito estadual surge em São Paulo, a Fundação de Amparo à Pesquisa do Estado de São Paulo (FAPESP) em 1960 (CNPq, 2019). Dessa forma, Balbachevsky (2010) destaca a criação da Fundação de Amparo à Pesquisa do Estado de São Paulo (FAPESP), em 1964, devido ao seu papel expressivo no fomento à pesquisa, operando em linhas similares às do CNPq, representando uma base de apoio importante para sustentar o desenvolvimento diferenciado do sistema de pesquisa. Assim, na década de 60, ocorreu um importante reforço às atividades de implantação de infraestrutura de pesquisa, principalmente devido à criação do FNDCT, em 1969, o que nas décadas seguintes desencadearam suporte para ações mais efetivas (CNPq, 2019).

Além da criação de instituições, ao longo desse período, alguns planos governamentais também foram desenvolvidos. Dentre eles, o Programa Estratégico de Desenvolvimento (PED), entre os anos de 1968 e 1970, definindo o desenvolvimento científico e tecnológico como objetivo da política governamental. Foi também pioneiro ao utilizar uma abordagem sistêmica, apontando para um Sistema Nacional de Desenvolvimento Científico e Tecnológico (SNDCT). Aliás, o SNDCT foi criado em 1972, e submetido à coordenação do Conselho Nacional de Desenvolvimento Científico e Tecnológico, criado em 1974 (FREITAS, 2014; OLIVEIRA, 2016).

Neste ínterim, ainda foram criados o Plano Nacional de Desenvolvimento (PND) e o Plano Básico de Desenvolvimento Científico e Tecnológico (PBDCT), que continha diretivas sobre pesquisa nas diversas áreas do conhecimento; criação de programas setoriais específicos; e,

Revista Eletrônica do Alto Vale do Itajaí - REAVI, v.08, nº 13, p. 073-083, dez 2019 ISSN: 23164190, DOI 10.5965/2316419008112019073 


\section{REAVI}

propunha aumento da interação do SNDCT com o setor privado (BALBACHEVSKY, 2010; FREITAS, 2014).

A década de 80 apresentou poucas mudanças significativas na configuração da área de C,T\&I no Brasil. Por exemplo, o PBDCT III que contemplava os anos de 1980 a 1985 foi descontinuado. Devido à configuração econômica da época, agências de fomento e comunidades de pesquisa perceberam a oportunidade em acessar recursos financeiros junto a agências internacionais para dar continuidade às suas pesquisas. Isto resultou na criação do Programa de Apoio ao Desenvolvimento à Ciência e Tecnologia (PADCT) (BALBACHEVSKY, 2010; FREITAS, 2014). Esse processo começou a mudar com o fim da ditadura militar, e com o processo de redemocratização em 1985. Com a criação do então Ministério da Ciência e Tecnologia (MCT) pelo Decreto 91.146, em 15 de março do mesmo ano, passou a atuar nas áreas que antes eram da competência do CNPq. Assim, o CNPq retornou a restrita responsabilidade na área científica (OLIVEIRA, 2015).

Em 1988, foi promulgada a Constituição Federal do Brasil, que incluiu um capítulo exclusivo para a C\&T, até então inexistente em qualquer carta constitucional (FREITAS, 2014). De fato, a Constitucional Federal de 1988 é um marco para o setor por balizar legalmente implantações de políticas públicas voltadas para a área de C\&T. Estabeleceu também, diretrizes para promoção do desenvolvimento científico e tecnológico e colocou-o na rota do governo federal (BALBACHEVSKY, 2010).

Os anos 90 apresentaram um novo cenário econômico para o país e também novos desafios para a área de $\mathrm{C}, \mathrm{T} \& \mathrm{I}$. Dessa forma, houveram três iniciativas para a reforma do setor: a negociação da terceira fase do PADCT, as reformas do CNPq e da FINEP (BALBACHEVSKY, 2010). Além disso, o apoio aos instrumentos de fomento e as diretrizes da Política Nacional de Ciência, Tecnologia e Inovação, como a continuação do Programa de Apoio ao Desenvolvimento Científico e Tecnológico (PADCT) II e III e o fortalecimento da pesquisa científica e tecnológica (FREITAS, 2014). O PADCT III foi elaborado após uma longa negociação e incorporou ao final boa parte dos novos instrumentos no âmbito das agências executoras do programa: FINEP, CNPq e CAPES (BALBACHEVSKY, 2010).

Também foram criadas Secretarias de C\&T e instalou-se uma rede de Institutos Nacionais de Ciência e Tecnologia, que substituíram os Institutos do Milênio. Ainda, foi elaborado um plano nacional de C\&T, como eixo orientador da política de Estado (FREITAS, 2014). A FINEP, por sua vez, lançou dois instrumentos no final da década de 90: o Programa de Apoio aos Núcleos de Excelência (PRONEX) e os Fundos Setoriais.

O PRONEX, criou instrumentos para sustentar núcleos de excelência científica no país. Os Fundos Setoriais, foram criados para fortalecer a pesquisa em setores tecnológicos estratégicos, tais como, energia elétrica, siderurgia, aeronáutica e petróleo. Ao todo, existem 16 fundos setoriais, 14 específicos e 2 transversais (BALBACHEVSKY, 2010).

\subsection{Século XXI e a Inovação como centro das políticas públicas brasileiras}

A virada do século representou grandes mudanças para área de inovação. A partir da II Conferência Nacional de Ciência, Tecnologia e Inovação, em 2001, o Brasil passou a incorporar o termo, agora denominando, Política de Ciência, Tecnologia e Inovação. A III Conferência Nacional de Ciência, Tecnologia e Inovação, que ocorreu em 2005, colocou maior ênfase no potencial da inovação para o desenvolvimento da indústria nacional; para dinamização das economias regionais, e; superação de situações de desigualdades (BALBACHEVSKY, 2010).

Revista Eletrônica do Alto Vale do Itajaí - REAVI, v.08, n 13, p. 073-083, dez 2019 ISSN: 23164190, DOI 10.5965/2316419008112019073 


\section{REAVI}

Em 2004, houve a aprovação da lei de inovação (lei $n^{\circ} 10.973$ ), alterada recentemente em 2016 pela lei $\mathrm{n}^{\circ} 13.243$ e regulamentada pelo decreto $\mathrm{n}^{\circ} 9.283$, de 7 de fevereiro de 2018 e a lei do Bem em 2005 (lei no 11.196). A lei da inovação foi criada para fornecer mecanismos para facilitar a circulação de pesquisadores entre as instituições de pesquisa e as empresas e, a promoção da cooperação universidade-empresa (BALBACHEVSKY, 2010; FREITAS, 2014). Em 2016 foi aprovada a lei $\mathrm{n}^{\circ} 13.243$ e o decreto $\mathrm{n}^{\circ} 9.283$ que regulamente a lei em 2018 , alterando a lei de 2004.

A lei $n^{\circ} 10.973$, visa melhorar a parceria entre Institutos de Ciência e Tecnologia com as empresas privadas, por meio da redução de pontos críticos de insegurança jurídica, além de esclarecer sua aplicação e operacionalização e, fortalece ferramentas de estímulo à participação de ICT's em atividades de inovação associadas ao segmento produtivo. Reduz, portanto, a burocracia envolvida em atividades de pesquisa científica, com a finalidade de proporcionar uma melhor articulação entre agentes de pesquisa com as empresas que realizam atividades inovadoras (BRASIL, 2018). Segundo Rauen (2016), entre as mudanças impostas, destacam-se: a formalização das ICT's privadas; a ampliação do papel dos núcleos de inovação tecnológica (NIT's); a diminuição de entraves para a importação de insumos para P\&D; a formalização das bolsas de estímulo à atividade inovativa, entre outros.

Desta forma, o Estado considera que a inovação pode ocorrer por meio de apoio a arranjos territoriais, formando ecossistemas de inovação, ou incentivo à interação entre os atores do Sistema de Inovação Brasileiro. Aliás, o decreto institucionaliza o Sistema Nacional de Ciência, Tecnologia e Inovação sob o regime de colaboração entre os estados da nação (BRASIL, 2018). A figura 1, apresenta os principais atores do sistema de inovação brasileiro, atualmente.

Figura 1 - Principais atores do sistema nacional de inovação do Brasil
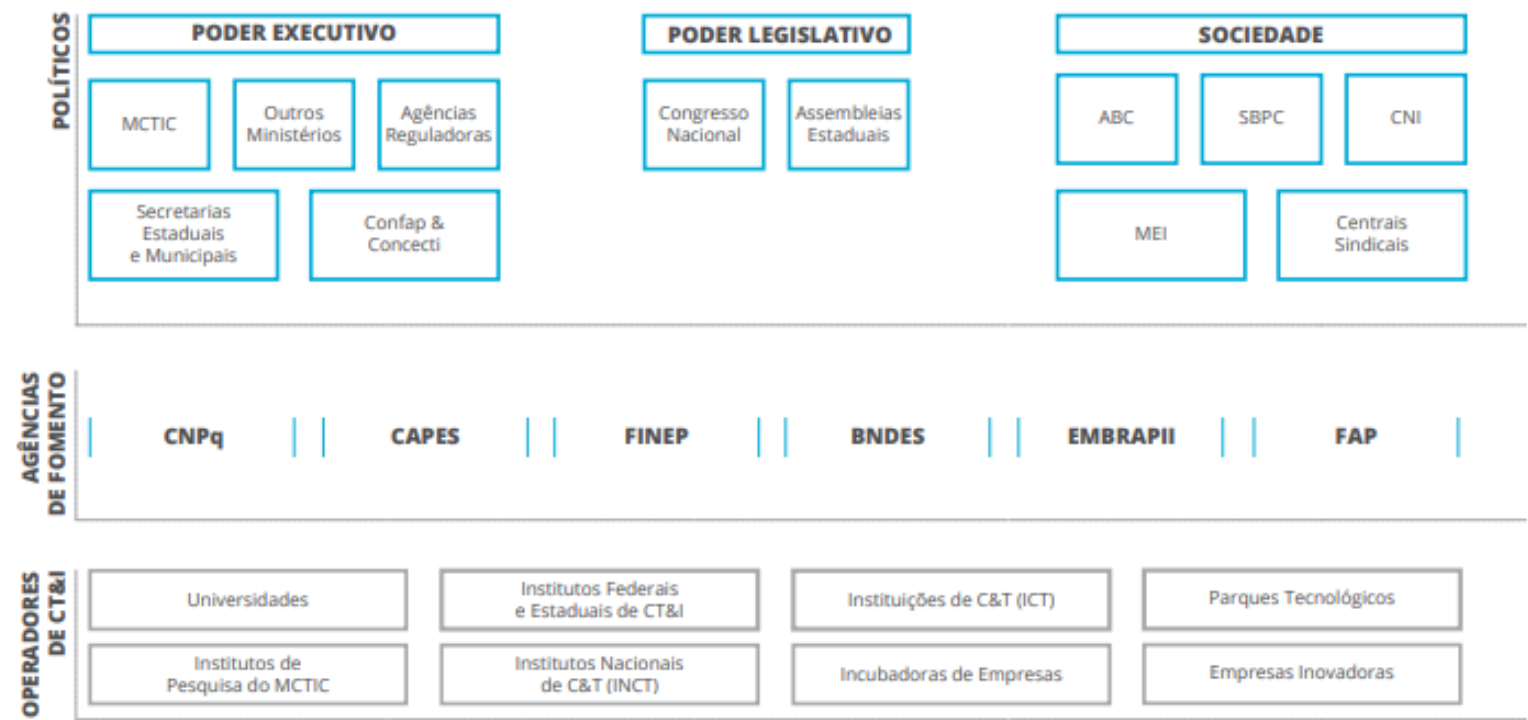

Fonte: Brasil (2016)

Por fim, decreto $\mathrm{n}^{\circ} 8.877$, de 18 de outubro de 2016, alterou as competências do Ministério da Ciência, Tecnologia, Inovação e Comunicações. Tais quais, ficou sob sua responsabilidade a política nacional de telecomunicações; política nacional de radiodifusão; serviços postais, telecomunicações e radiodifusão; políticas nacionais de pesquisa científica e tecnológica e de

Revista Eletrônica do Alto Vale do Itajaí - REAVI, v.08, n 13, p. 073-083, dez 2019 ISSN: 23164190, DOI 10.5965/2316419008112019073 


\section{REAVI}

incentivo à inovação; planejamento, coordenação, supervisão e controle das atividades de ciência, tecnologia e inovação; política de desenvolvimento de informática e automação; política nacional de biossegurança; política espacial; política nuclear; controle da exportação de bens e serviços sensíveis; articulação com os Governos dos Estados, do Distrito Federal e dos Municípios, com a sociedade civil e com órgãos do Governo federal para estabelecimento de diretrizes para as políticas nacionais de ciência, tecnologia e inovação (BRASIL, 2016b).

Outro fato recente que merece atenção, foi a criação e continuidade pelo Ministério de Ciência, Tecnologia e Inovação (MCTI), em 2012, da Estratégia Nacional de Ciência, Tecnologia e Inovação (ENCTI). Atualmente, está em vigência a segunda edição para o período de 2016-2022. O objetivo da Estratégia é nortear ações que contribuam para o desenvolvimento nacional, por meio de iniciativas que valorizem o avanço do conhecimento e da inovação (BRASIL, 2016). Assim, os pilares fundamentais do sistema de inovação do Brasil, considerados pelo documento, são: pesquisa; infraestrutura; financiamento; recursos humanos; e, inovação. Portanto, é a partir do fortalecimento desses pilares que se pode promover a expansão, consolidação e integração do sistema nacional de inovação.

A ANPEI a partir de um estudo com 237 atores brasileiros, criou o mapa do sistema nacional de inovação, apresentado na figura 2. O mapa representa os fluxos de interações entre as esferas e suas ocorrências.

Figura 2 - Mapa do Sistema Brasileiro de Inovação.

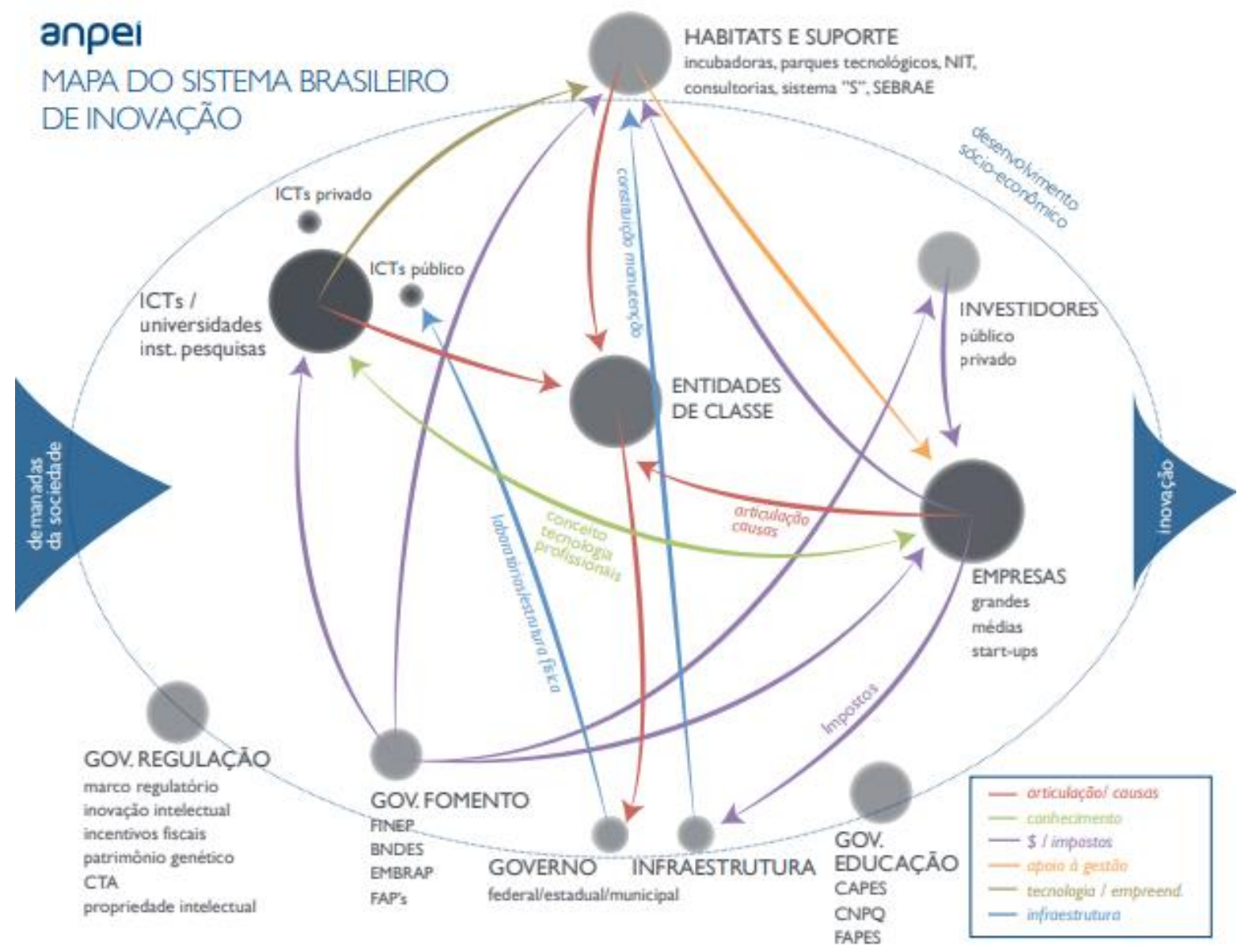

Fonte: ANPEI (2014)

Revista Eletrônica do Alto Vale do Itajaí - REAVI, v.08, n 13, p. 073-083, dez 2019 ISSN: 23164190, DOI 10.5965/2316419008112019073 


\section{REAVI}

Assim, ao analisar a imagem percebe-se um número considerável de interação saindo de atores do governo, por exemplo, fomento para empresas, investidores, habitats e suporte e ICTs. Assim, apenas entidades de classe não recebem fomento do governo.

A Figura 3 relata a interação e o nível de proximidade entre os atores do Sistema de Inovação do Brasil, e seus pontos de intersecção.

Figura 3 - Grau de interação entre os atores.

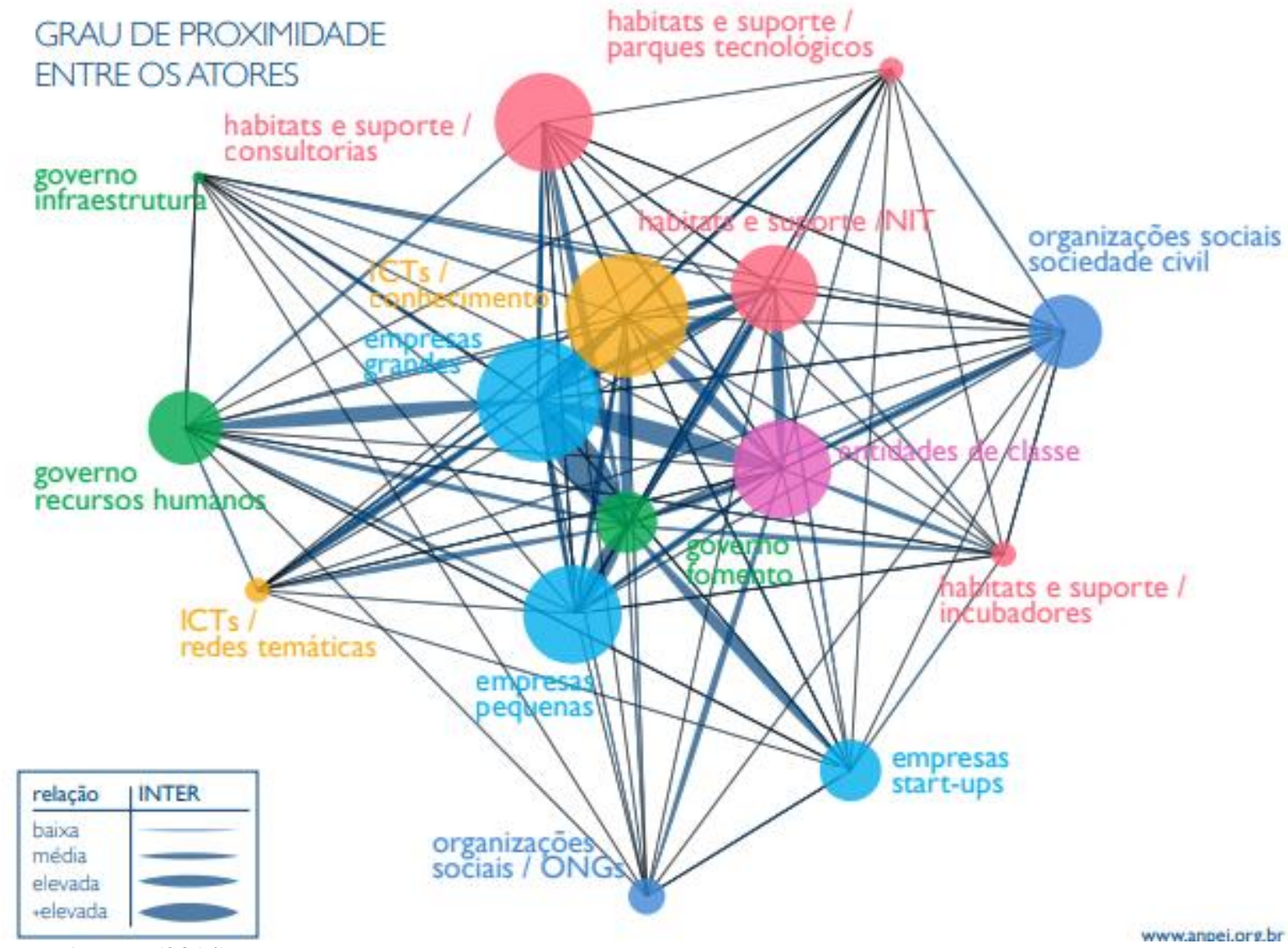

Fonte: ANPEI (2014).

Nota-se que quanto maior for a interação, mais espessa é a linha que liga os atores, assim como maior é a esfera. Esse mapa demonstra, por exemplo, que as grandes empresas estão no centro do mapa, com o maior número de interações, juntamente com Institutos de Ciência e Tecnologia e Conhecimento. Além disso, a linha que liga o fomento público á grandes empresas é a maior interação existente do mapa. Isso demonstra que as grandes empresas são capazes de articular com vários atores diferentes do Sistema, e de captar recursos de fomento público, sendo as maiores beneficiadas. Por outro lado, habitats e suporte como incubadoras e parques tecnológicos possuem pouca interação, o que pode explicar o porquê das empresas startups serem aquelas que tenham menor interação em relação as grandes e médias.

Revista Eletrônica do Alto Vale do Itajaí - REAVI, v.08, n 13, p. 073-083, dez 2019 ISSN: 23164190, DOI 10.5965/2316419008112019073 


\section{REAVI}

\section{Conclusão}

O presente artigo tem como objetivo, apresentar a evolução do sistema de inovação brasileiro, sua configuração atual e suas interações. Como apresentado, o Brasil tem mais de um século desde a criação das primeiras instituições de ciência e tecnologia. A evolução da criação destas instituições, estruturou no Brasil um sistema nacional de inovação. Esta teoria, difundida em todo o mundo, balizou a criação de diversas políticas de C,T\&I. Desta forma, entre as principais instituições nacionais de fomento a inovação são o CNPq e a CAPES, atores relacionados ao conhecimento. Outro ator fundamental é a FINEP, maior financiadora de estudos e projetos do país, que fomenta desde $\mathrm{P} \& \mathrm{D}$ nas empresas até infraestrutura em instituições de ciência e tecnologia.

Além disso, programas e legislações foram criadas para incentivar aquilo que é fundamental num sistema de inovação, que é a interação, e, também fazem parte do hall de atores do sistema. Assim, o Brasil criou em 2004 a lei de inovação que foi atualizada em 2016 e regulamentada em 2018. Além da legislação com objetivo de aproximar ICTs e empresas e desburocratizar as relações públicas e privadas, foi criada uma estratégia (ENCTI) que visa colocar o país em outro patamar de desenvolvimento tecnológico em médio e longo prazo. O desafio é superar anos de atraso em relação aos países em desenvolvimento.

Também foi possível, visualizar o quadro dos principais atores do sistema de inovação do Brasil, no qual fazem parte atores políticos, agentes de fomento e operacionalizadores de C,T\&I. Além disso, a partir do estudo da ANPEI, foi possível visualizar os fluxos e o grau de interações entre as instituições. Portanto, compreende-se que o maior número de interação ocorre entre grandes empresas, governo (principalmente, fomento) e ICT's.

Portanto, o fortalecimento dessas instituições e principalmente a descentralização de suas ações é fundamental, não apenas para reduzir o atraso tecnológico, mas também, reduzir as desigualdades regionais. Estudos futuros podem analisar como a ENCTI está sendo executada.

\section{REFERÊNCIAS}

ANPEI. Associação Nacional de Pesquisa e Desenvolvimento das Empresas Inovadoras. Mapa do Sistema Brasileiro de Inovação. Comitê Interação ICT - Empresa. Comitê de Fomento à Inovação. São Paulo, 33f. 2014.

ASHEIM, B.; GRILLITSCH, M.; TRIPPL, M. Regional Innovation Systems: Past - Presence Future, Circle: Papers in Innovation Studies, Oslo, v. 36, 2015.

ASHEIM, B.; SMITH, H. L.; OUGHTON, C. Regional Innovation Systems: Theory, Empirics and Policy, Regional Studies, [s.1.], v. 45, n. 7, p. 875-891, 2011.

BALBACHEVSKY, E. Processos Decisórios em política científica, tecnológica e de inovação no Brasil: nova geração da política de ciência, tecnologia e inovação. Brasília: CGEE-MCTI, 2010.

BRASIL. Decreto $\mathbf{n}^{\mathbf{0}}$ 8.877, de 18 de outubro de 2016. 2016b. Disponível em: <encurtador.com.br/fgqyW>. Acesso em: 20 jul. 2019.

Revista Eletrônica do Alto Vale do Itajaí - REAVI, v.08, n 13, p. 073-083, dez 2019 ISSN: 23164190, DOI 10.5965/2316419008112019073 


\section{REAVI}

BRASIL. Decreto no 9.283, de 7 de fevereiro de 2018, 2018. Disponível em: http://bit.ly/2Ou0K32. Acesso em: 20 jul. 2019.

BRASIL. Ministério da Ciência Tecnologia e Inovação (MCTI). Estratégia Nacional de Ciência Tecnologia e Inovação 2016 - 2022. Brasília, 2016. Disponível em: <encurtador.com.br/bcfv6>. Acesso em: 30 jun. 2019.

CAPES. História e missão. 2019. Disponível em: 〈http://bit.ly/2KeapFt〉. Acesso em: 20 jul. 2019.

CNPQ. Anos 60. 2019. Disponível em: 〈http://bit.ly/2ZwqH2I>. Acesso em: 20 jul. 2019. COOKE, P. Regional Innovation Systems: competitive regulation in the new europe. Geoforum, 23, p. 365-382, 1992.

FINEP. Histórico. 2019. Disponível em: 〈http://bit.ly/2LPL1Jq>. Acesso em: 20 jul. 2019.

FINEP. Histórico. 2019b. Disponível em: 〈http://bit.ly/2LPL1Jq>. Acesso em: 20 jul. 2019.

FREEMAN, C. The 'National System of Innovation'in historical perspective. Cambridge Journal of economics, v. 19, n. 1, p. 5-24, 1995.

FREITAS, R. L. O Processo de Acompanhamento e Avaliação das Transferências Voluntárias de Recursos do CNPq em Parceria com as FAP. Mestrado Profissional em Gestão e Política de Ciência Tecnologia e Inovação, Universidade de Brasília, 2014.

GODIN, B. Innovation: a conceptual history of an anonymous concept. Project on the Intellectual History of Innovation, Quebec, v. 21, n. 1, p. 1-36, 2015.

LARANJA, M.; UYARRA, E.; FLANAGAN, K. Policies for science, technology and innovation: Translating rationales into regional policies in a multi-level setting. Research Policy, [s.1.], v.37, n.5, p. 823-835, 2008.

LÜDKE, M.; ANDRÉ, M. Pesquisa em educação: abordagens qualitativas. São Paulo: EPU, 1986.

LUNDVALL, B. National innovation systems. Pinter, London, 1992.

NELSON, R. R. National innovation systems: a comparative analysis. Oxford University Press on Demand, 1993.

OLIVEIRA, J. Ciência, tecnologia e inovação no Brasil: poder, política e burocracia na arena decisória. Revista de Sociologia e Política, v. 24, n. 59, p. 129-147, 2016.

RAUEN, C. V. O Novo Marco Legal da Inovação no Brasil: O que muda na relação ICTEmpresa? Radar, v. 1, n. 41, p. 21-35, 2016.

Revista Eletrônica do Alto Vale do Itajaí - REAVI, v.08, n 13, p. 073-083, dez 2019 ISSN: 23164190, DOI 10.5965/2316419008112019073 


\section{REAVI}

TANG, M.; BASKARAN, A.; YAN, H.; MUCHIE, M. Strengthening regional integration/cooperation with the Neighbourhood System of Innovation conceptual framework: the case of China and ASEAN. Asian Journal of Technology Innovation, [s.1.], v. 23, n. 2, p. 205-229, 2015.

ZARAYCHENKO, I.; SHINKEVICH, A.; SHVETSOV, M.; ERDYNEYEVA, K.; BORDONSKAYA, L.; PERSIDSKAYA, A.; ROZHKOVA, S.; AFANASYEV, A. Innovation Networks Modeling Within the Concept of Open Innovations. International Journal of Economics and Financial Issues, v.6, n.1, p. 192-198, 2016.

Revista Eletrônica do Alto Vale do Itajaí - REAVI, v.08, n 13, p. 073-083, dez 2019 ISSN: 23164190, DOI 10.5965/2316419008112019073 